\title{
Sociedad Civil y combate a la pobreza: Impacto nutricional y económico de la intervención de Banco de Alimentos de Hermosillo en la comunidad de Pesqueira, Sonora, México
}

\author{
Ricardo López Salazar \\ Centro de Investigación en Alimentación y Desarrollo, Campus Hermosillo, Sonora, \\ México. \\ Email: ricardolopezsalaz@gmail.com
}

\section{Isabel Ortega}

Centro de Investigación en Alimentación y Desarrollo, Campus Hermosillo, Sonora, México.

Email: iortega@ciad.mx

\section{Sergio Sandoval}

Centro de Investigación en Alimentación y Desarrollo, Campus Hermosillo, Sonora, México.

Email: ssandoval@ciad.mx

Resumen: Una de las organizaciones de la sociedad civil que ha adquirido gran relevancia en el combate a la pobreza alimentaria en el estado de Sonora es el Banco de Alimentos de Hermosillo (BAH). Su objetivo es combatir el hambre y la desnutrición mediante la distribución de alimentos a zonas marginadas. Pesqueira, es una de las localidades en situación crítica de pobreza alimentaria y que ha sido beneficiada por los apoyos que otorga el BAH. Sin embargo, a pesar de que esos apoyos son reconocidos como una ayuda importante en términos de asistencia alimentaria, se desconoce objetivamente su impacto nutricional y económico. La metodología consistió en la tipificación de las canastas alimentarias (CA) durante un periodo de cuatro meses para contrastar su aporte nutricional y su valor en el mercado. Los resultados son los siguientes: de acuerdo a la composición nutrimental de la CA, es posible observar que sólo cubre el $20 \%$ de la ingestión diaria recomendada. En términos económicos, las CA han contribuido a que la población beneficiaria reduzca sus niveles de pobreza alimentaria en un $40 \%$, aunque persiste la pobreza en términos absolutos.

Palabras clave: Banco de alimentos de Hermosillo, pobreza alimentaria, Pesqueira, canastas alimentarias, aporte nutrimental 


\title{
Civil Society and the fight against poverty: nutritional and economic impact of the intervention of the Food Bank of Hermosillo in the community of Pesqueira, Sonora, México
}

\begin{abstract}
One of the organizations of the social society, which has been acquiring a high relevance in the fight against food poverty is The Food Bank of Hermosillo (BAH), located in the Mexican state of Sonora. The goal of BAH is to fight hunger and undernourishment by the distribution of food to marginalized zones. One of the localities that has been benefitted by BAH is Pesqueira, which is in a critical situation of malnutrition and poverty. Nevertheless, although the nourishing supports that the bank in Pesqueira grants are recognized as an important aid in terms of nourishing attendance, the nutritional and economic impact of these supports is not known objectively. The methodology applied consisted in the standardization of the baskets during a period of four months to contrast its nutritional contribution and the value of the same in the market. The results are the following: according to the nutrimental composition of the basket, it is possible to observe that it only covers $20 \%$ of the recommended daily ingestion. In economic terms, the CA has contributed to the degree that the beneficiary population reduces its levels of nourishing poverty in $40 \%$, although poverty in absolute terms persists.
\end{abstract}

Key words: Food Bank of Hermosillo, food poverty, Pesqueira, food baskets, nourishing support

\section{Sociedade civil e luta contra a pobreza: impacto nutricional e econômica da intervenção do Banco de Alimentos de Hermosillo na comunidade de Pesqueira, Sonora, México}

Resumo: Uma das organizações da sociedade civil, que foram adquiridos mais relevante na luta contra a pobreza alimentar é o Banco de Alimentos de Hermosillo (BAH), localizada no estado mexicano de Sonora. O objetivo da BAH é a luta contra a fome ea desnutrição através da distribuição de alimentos às zonas marginalizadas. Uma das localidades que foram beneficiadas pelo BAH é Pesqueira, que está em situação crítica de pobreza alimentar. No entanto, embora o suporte nutritivo que o banco em bolsas de Pesqueira são reconhecidas como uma ajuda importante em termos de frequência alimentar, o impacto nutricional e econômica desses apoios não é conhecido objetivamente. A metodologia consistiu na padronização das cestas durante um período de quatro meses para resistir à sua contribuição nutricional eo valor da mesma no mercado. Os resultados são os seguintes: de acordo com a composição da cesta Nutrimental, é possível observar que ele só cobre $20 \%$ com a ingestão diária recomendada. Em termos econômicos, o CA ter contribuído para que a população beneficiária reduz seus níveis de pobreza alimentar em $40 \%$, apesar de persistir a pobreza em termos absolutos.

Palavras-chave: Banco Alimentar de Hermosillo, pobreza alimentar, Pesqueira, cestas básicas, suporte nutritivo 


\section{Introducción}

Durante gran parte del siglo anterior, el Estado dominó el escenario económico, político y social imperante en los países desarrollados y emergentes, para posteriormente apartarse y cederle la iniciativa al mercado en aras de recuperar la estabilidad y crecimiento económico perdido durante los setentas. A partir de la adopción del modelo centrado en el mercado como base de crecimiento, y la profundización de fenómenos como la globalización, y la desregulación comercial, se llegó a pensar que la resolución de los problemas sociales se gestaría a partir de la entrada a un nuevo ciclo de crecimiento económico sostenido. Sin embargo, las ambivalencias respecto a los costos y beneficios del modelo de mercado, han catalizado la irrupción de nuevos actores y colectividades que han tomado un papel preponderante en la esfera pública.

En este tenor, la sociedad civil organizada como un espacio de acción que va más allá de la lógica y las pautas que marcan la relación Estado-mercado, ha significado un espacio revitalizador para discutir, concertar e implementar iniciativas dirigidas a mitigar y/o solucionar problemas específicos de la población. En este documento se analiza una de estas organizaciones de la sociedad civil, conocida como Banco de Alimentos de Hermosillo (BAH), que opera en el norteño estado de Sonora, México, y que tiene como objetivo combatir el hambre y la desnutrición. En particular, el BAH, trabaja como un puente entre donadores de alimentos y beneficiarios, es decir, acopia, selecciona y distribuye alimento perecedero y no perecedero y lo hace llegar en forma organizada a instituciones de asistencia social y comunidades marginadas de zonas urbanas, rurales e indígenas. Un breve análisis de sus impactos nutricional y económico sirve de soporte para sostener que la participación de la sociedad civil es fundamental en el diseño e implementación de políticas públicas locales de combate a la pobreza y lograr el desarrollo desde el lugar donde se padecen estos problemas.

En los últimos años el BAH, ha incrementado su presencia hacia otros municipios del estado, sobre todo de aquellos cercanos a Hermosillo (capital del estado de Sonora). Uno de esos municipios que ha recibido cobertura del BAH es San Miguel de Horcasitas, el cual se encuentra en una situación crítica en materia alimentaria, ya que cerca del $50 \%$ del total de su población se encuentra en pobreza alimentaria (Coneval, 2008). En particular, los apoyos alimentarios del BAH en San Miguel de Horcasitas se han canalizado a la comunidad de Pesqueira.

A pesar de que los apoyos alimentarios que otorga el BAH en Pesqueira son reconocidos como una ayuda importante en términos de asistencia alimentaria, se desconoce el impacto nutricional y económico de dicha ayuda. Por ello, el objetivo del presente trabajo es presentar parte de los resultados obtenidos mediante el trabajo de campo para conocer una aproximación de esos impactos en las familias beneficiarias. 
A partir de lo anterior, se realizan algunas sugerencias y recomendaciones en la conformación de las canastas alimentarias (CA) considerando los aportes nutricionales de sus componentes. Para lograr ayudar a las personas a adquirir alimentos con la finalidad de que mejoren sus aspectos nutricionales, y con ello contribuir a elevar su calidad de vida y sus posibilidades de desarrollo. De esta manera, se acentúa la importancia que siguen ganando las organizaciones de la sociedad civil en el campo de lo público, y en particular la contribución que estas realizan a combatir un problema tan complejo como la pobreza alimentaria.

La metodología se dividió en tres partes: tipificación de la canasta otorgada por el banco en la comunidad, análisis nutricional y análisis económico. Para la elaboración del análisis nutricional se procedió a la tipificación de las canastas alimentarias que otorga el BAH (Allen, 2001). Para ello, se analizaron las frecuencias en que aparecen cada uno de los componentes de las CA otorgadas por el BAH a la comunidad de Pesqueira, en un período de cuatro meses (enero-mayo del 2009). Adicionalmente, se agruparon todos los alimentos de las CA en tres clasificaciones: 1) alimentos básicos, 2) alimentos secundarios y, 3) alimentos periféricos (FAO, 2002). Para la realización del análisis económico, se obtuvo el costo de mercado de las CA tipificadas y se comparó con el costo de la Canasta Normativa Alimentaria (CNA) fijada por el gobierno mexicano, con el propósito de identificar, en términos de canastas, el aporte económico del BAH a las familias beneficiarias de Pesqueira. La muestra consistió en seleccionar a 21 familias que además de recibir el apoyo del BAH, tuvieran como integrante al menos a un niño de de 3-7 años, esto porque ellos son más susceptibles de padecer problemas relacionados con la alimentación. Por lo tanto, en especial se analiza la aportación de las canastas en dos micro nutrimentos vitamina A y Hierro, los cuales son esenciales para el crecimiento y desarrollo de los infantes.

El documento se estructura de la siguiente manera. En la primera parte se discute la participación de la sociedad civil en las políticas públicas para lograr el desarrollo local. La segunda parte aborda de manera más específica la metodología utilizada para la elaboración del documento. En la tercera parte se presentan los resultados del impacto nutricional, en la cuarta parte se analizan los aspectos económicos que genera el apoyo del BAH en Pesqueira. Finalmente se presentan algunas consideraciones y reflexiones finales.

\section{El desarrollo local a partir de la participación de la socie- dad civil en las políticas públicas}

\section{El concepto de desarrollo local}

A pesar de que la noción de desarrollo local se vislumbró desde principios del siglo pasado por diversos académicos como Marshall y su idea de los distritos industriales como fuente de desarrollo, ésta permane- 
ció inactiva en las principales corrientes académicas de la época ante la emergencia del keynesianismo y del gran monopolio que ejerció el Estado en la economía desde los 20’s hasta los 70’s. Sin embargo, con el devenir del siglo y el desmoronamiento de la economía planificada que derivó en la emergencia del mercado como el vértice central sobre el cual giraría una nueva época sostenida de bienestar económico y ante los nuevos problemas que este modelo ha generado, la idea del desarrollo local comienza a tomar fuerza desde diversos círculos académicos para enfrentar estos retos.

Sin embargo, existen notables controversias acerca de lo que realmente es el desarrollo local y su emergencia como corriente teórica de importancia. Según Boisier, por desarrollo local debe entenderse al proceso capilar, local, endógeno, continuo o discontinuo sobre el territorio, que a diferencia del crecimiento que puede ser inducido desde arriba o desde abajo, responde a particularidades concretas sólo atribuibles a una realidad específica. También señala que sobre la visión de desarrollo local persisten múltiples definiciones conceptuales como desarrollo endógeno, desarrollo económico local, desarrollo descentralizado, entre otros, que abonan a la confusión del mismo y, por tanto, derivan en la dificultad de su implantación como un concepto homogéneo (Boisier, 2000: 21).

Por otro lado, Vázquez Barquero apunta que el desarrollo local es aquel que se logra mediante la concertación de una serie de actores locales que tienden a desarrollar sus capacidades hacia la generación de iniciativas que logren incrementar el bienestar de las personas. De esta definición se puede inferir que el desarrollo no es un proceso exclusivo de un actor en particular así como tampoco polariza a los actores públicos y privados, sino que más bien se aboca a generar convergencias y uniones que permiten la emergencia de iniciativas concretas a una localidad o a un territorio (Vazquez Barquero, 2005.)

Entonces, el desarrollo local en sustancia y acción implica la emergencia de actores públicos y privados que contribuyen a la utilización de las potencialidades internas del territorio y/o localidad en aras de incrementar el bienestar de las personas. La simple aproximación anterior es por sí misma más compleja, ya que habría que poner sobre la mesa una serie de aspectos que parecen quedar sueltos, como los grados de responsabilidad de los actores públicos y privados en el proceso, los tipos de coordinación, el rol y la importancia que juegan las instituciones locales, por mencionar algunas. Clarificar estos aspectos abona a una mejor comprensión del desarrollo local, sin embargo, la intención del presente trabajo no es analizar dicho concepto, por lo cual no se profundizará en su análisis.

\section{El desarrollo local y su vinculación con la sociedad civil}

En las últimas décadas, el concepto de desarrollo local está estrechamente vinculado a la participación de la sociedad civil organizada ya que esta ha comenzado a intervenir activamente en todos los ámbitos que le son posibles, hecho que significa un replanteamiento del papel de todos los 
actores sociales en una sociedad que evoluciona a pasos agigantados. Durante la primera mitad del siglo pasado el Estado representaba el actor central a partir del cual se generaban las principales iniciativas de crecimiento y desarrollo, sin embargo en las últimas dos décadas transfirió muchas de sus responsabilidades al mercado y a la misma sociedad civil, además de que esta última ha ido avanzando en la conquista de espacios que estaban bajo el control del gobierno, principalmente en cuestiones de desarrollo.

La emergencia de los actores sociales organizados que ocupan espacios desatendidos por el Estado y el mercado, es un elemento que bien se puede tomar como un indicador del desarrollo de cualquier sociedad. Debido a que pone de manifiesto la capacidad de las personas para hacerle frente a escenarios adversos con una lógica de solidaridad que le corresponde a sociedades modernas y organizadas. Es por ello que Diamond (2005), señala que la sociedad civil se presenta como un espacio abierto a la construcción de formas asociativas independientes del control gubernamental y que ejerce influencia en el diseño de las políticas gubernamentales.

En este punto resulta indispensable reflexionar acerca de la sociedad civil en su acepción más pura y llana, tal como lo menciona Diamond quien define a la sociedad civil como:

“El reino de la vida social organizada que es voluntaria, autogeneradora, autónoma frente al Estado y protegida por el orden legal. La sociedad civil es diferente de la sociedad en general porque la primera involucra a los ciudadanos que actúan colectivamente en la esfera pública” (Diamond, 2005:135.)

La definición aportada por Diamond posibilita vislumbrar tres aspectos claves para comprender de mejor forma de qué estamos hablando cuando nos referimos a la sociedad civil. En primer lugar, pone de manifiesto la independencia y autonomía que ésta debe tener respecto al Estado, es decir, si bien puede interactuar en áreas de interés compartidas con el Estado, ello no debe de provocar alguna clase de subordinación hacia este último, ya que pasaría a formar parte de los grupos burocráticos que comúnmente se conforman al interior de los aparatos gubernamentales y que obedecen a otros intereses asintóticos a los perseguidos por la sociedad civil.

En segundo lugar, para que la sociedad civil pueda desenvolverse libremente tiene que estar respaldada por la presencia efectiva del estado de derecho. Es decir, cobijada bajo el orden legal correspondiente, ya que la incertidumbre y la carestía de un estado de derecho efectivo disminuyen el interés de las personas y sus asociaciones por involucrarse en la esfera pública.

En tercer lugar, a pesar de que la sociedad civil se desenvuelve en una sociedad concreta, la cual invariablemente se compone por personas, 
la diferencia entre una y otra radica en el interés que manifiestan ciertos grupos en participar de manera colectiva en la esfera pública. Los grupos organizados con interés en participar, y también catalogados en el lenguaje anglosajón como stakeholders vienen a darle dirección a las iniciativas surgidas en el seno de un grupo de colectividades que manifiesta interés particular en alguna área específica.

Aquí conviene profundizar un poco más en los prerrequisitos que deben de conjugarse para facilitar la emergencia y consolidación de la sociedad civil, ya que sería un error suponer que el estado de derecho, la autonomía y la diferenciación de ciertos grupos o colectivos dentro de una sociedad se generan espontáneamente. Según Mouzelis, los elementos básicos que supone el apuntalamiento de la sociedad civil son: 1) la existencia de un estado de derecho que efectivamente proteja a los ciudadanos frente a las arbitrariedades de los funcionarios públicos; 2) la sólida presencia de organizaciones no-estatales capaces de vigilar eventuales abusos de poder de parte de quienes controlan las orientaciones de la administración pública y la coerción; 3) la existencia de un pluralismo balanceado entre los intereses de la sociedad civil, tal que ninguno de esos intereses pueda establecer un dominio absoluto (Mouzelis, 1991).

La sociedad civil por si misma tampoco representa la panacea que resuelve el colectivo de problemas sociales existentes, sin embargo al menos orienta los procesos hacia la consecución de logros específicos que pueden ser plenamente identificados por las personas. La materialización de las acciones que lleva a cabo la sociedad civil depende de contextos específicos, así como de ambientes que la favorecen o la desincentivan, tal como se señaló con anterioridad. Curill (2006) destaca que esta participación tiene dos expresiones sociales: la defensa de intereses particulares (colectivos y difusos) y la defensa de concepciones alternativas sobre el interés público.

Si bien es cierto que la sociedad civil organizada ha adquirido un papel relevante, también hay que reconocer que están limitados en cuanto a los recursos de que disponen para atender diversos problemas que el Estado no atiende. Así, las ONG's juegan un papel cada vez mas importante en la vida de los pobres, pues las reformas políticas y económicas impulsadas en las últimas décadas han creado un proceso político que traduce las desigualdades económicas en desigualdades políticas y sociales (Holzner, 2007).

Según Aguilar (2008), las políticas públicas son resultado de un proceso social y político, que a veces es fluido y consensual. Donde los actores gubernamentales y sociales comparten información y aceptan los resultados del análisis del experto del gobierno, la academia o la consultoría. Mientras que otras veces es un proceso complejo, tenso, volátil, sacudido por discrepancias sobre los objetivos y acciones de las políticas, que obligan a negociaciones, ajustes, compensaciones entre los actores políticos y sociales interesados en la atención de un cierto 
asunto público y en la necesidad de echar a andar una cierta política en respuesta.

Al abordar la relación entre el desarrollo local y la participación ciudadana, es precisamente en el municipio porque es el orden de gobierno más cercano a la población, y donde a través del Ayuntamiento se debe construir un gobierno de participación ciudadana organizada. López (2007) indica que la participación social y comunitaria parten del reconocimiento de que existen problemas y necesidades que se tienen que resolver, independientemente de que se conciban o no como obligación del gobierno, y hacen uso de su derecho de participación como ciudadanos para resolverlos. Las diversas formas de participación de la ciudadanía y de la sociedad civil en general pueden contribuir al mejoramiento y perfeccionamiento de la democracia, sobre todo en el nivel de gobierno más cercano a la población. La necesidad que se tiene de hacer partícipe a la población es en la elaboración de las políticas públicas, siendo puntuales en cómo se deben elaborar y qué se entiende conceptualmente por el término, de qué manera su elaboración lleva consigo el consenso gobierno-ciudadanía. Además de conocer cómo con este binomio se pueden generar mejores alternativas de desarrollo para los habitantes de cada municipio, entrando en un proceso de gobernanza.

Fuente y Heiss definen a la sociedad civil como "el espacio intermedio situado entre el Estado y la familia, poblado por grupos o asociaciones organizados o separados del Estado y que disfrutan de cierta autonomía en relación a este último, y que son voluntariamente formados por miembros de la sociedad para proteger o ampliar sus intereses, valores o identidades" (Fuente y Heiss, 2006: 354). Además sostienen que la sociedad civil adquiere el papel preponderante como la única capaz de otorgar legitimidad democrática a procesos políticos y sociales, y de mediar entre lo económico y lo político, principalmente ahora que las políticas sociales están sujetas a la política económica.

Sin embargo, la sociedad civil no está libre de disputas políticas que sería importante sistematizar dado que los temas donde interviene no están libres de un debate ideológico mayor. Incluso, pueden estar relacionados con los partidos políticos. Tampoco buscan siempre el bien común. El impacto de un grupo especifico no solo dependerá de sus estrategias o del establecimiento de alianzas, sino también de la estructura y estrategia de las fuerzas que resisten el cambio.

No obstante ante estos obstáculos, es importante incluir la participación de la sociedad civil en el diseño e implementación de políticas públicas desde el ámbito local, pues es donde se perciben de manera más directa los problemas sociales, económicos y políticos resultantes de las desigualdades sociales y la desatención de otros órdenes de gobierno. La inclusión de diversos actores reduce las probabilidades de fracaso en el cumplimiento de los objetivos por la apatía u oposición de los actores locales y no gubernamentales. 


\section{Metodología}

\section{Tipificación de la Canasta Alimentaria}

Para determinar la composición de una Canasta Alimentaria estándar, se analizaron las frecuencias en que aparece cada uno de los componentes de las mismas, en un periodo de 4 meses y que fueran otorgadas en la comunidad de Pesqueira. Dicho de otra manera, analizamos los listados de $\mathrm{BAH}$ sobre los componentes de las canastas alimentarias otorgadas en la comunidad.

Lo anterior permitió hacer una distinción entre los alimentos que se entregaron con mayor y menor frecuencia a la comunidad. De igual manera se analizaron las proporciones (expresadas en cantidades - g/semana -) en que fueron entregados dichos alimentos, conduciéndonos a una segunda distinción entre alimentos entregados en mayor y menor proporción. Por último se agruparon todos los alimentos de las Canastas en tres clasificaciones: 1) alimentos básicos, 2) alimentos secundarios y, 3) alimentos periféricos. La determinación de la canasta alimentaria estándar se efectuó conjugando los alimentos que aparecieron más frecuentemente y en mayor proporción en cada estrato de clasificación de alimentos.

\section{La evaluación nutricional}

A partir de la tipificación de la canasta estándar otorgada por BAH en Pesqueira, se calculó el contenido promedio de energía y nutrimentos de todos los alimentos que conforman la canasta alimentaria otorgada por BAH. Para la elaboración de dicho cálculo se utilizó el diccionario de alimentos elaborado por el CIAD, AC.

Para el cálculo de los aportes de energía y nutrimentos de la canasta por hogar y per cápita, se dividieron las calorías y nutrimentos (en gramos) entre los 30 días que abarca la ayuda, así como entre el número de integrantes del hogar que recibieron el apoyo, para obtener la cantidad de calorías y nutrimentos diarios por persona.

Asimismo, se calculó la distribución de energía empleando el sistema de factor general de Atwater (Atwater y Woods, 1896, en FAO Food and nutrition paper 77), donde el aporte calórico es: carbohidratos (4 Kcal/ g), grasas (9 Kcal/g) y proteína (4 Kcal/g).

\section{El análisis económico}

Gracias a la tipificación de la CA se obtuvo el costo de mercado de cada bien en los últimos 4 meses en que fue entregado el apoyo de BAH en Pesqueira (febrero-mayo de 2009, tomando como referencia los precios establecidos por Profeco e INEGI). Los precios de cada bien se sumaron en conjunto para estimar el precio promedio que tendría la canasta alimentaria si los beneficiarios la adquirieran a precios de mercado. 
Adicionalmente, el costo de la CA se utilizó para la construcción de tres indicadores: primro, cambio en el nivel de gasto alimentario de los beneficiarios (CGA). Dicho indicador trata de mostrar las modificaciones en el gasto total en alimentación de los beneficiarios gracias al apoyo que les otorga el Banco. El supuesto central del indicador reside en que los beneficiarios aunque sea de manera indirecta (puesto que la canasta se recibe a un precio máximo de $\$ 25$ o incluso gratis en algunos casos) incrementan su gasto en alimentación gracias al subsidio del banco. La ecuación es la siguiente: $\mathrm{CGA}=$ Gasto alimentario de los beneficiarios + valor de la CA.

Segundo, participación de la Canasta Alimentaria de Banco de Alimentos en el ingreso de los beneficiarios (PCAI). Antes de recibir el apoyo del Banco, invariablemente todos los beneficiarios son sometidos a una encuesta socioeconómica que ayuda a la institución a seleccionar a las personas que son sujetas del apoyo. Así, el banco posee una estimación del nivel de ingresos de cada uno de los beneficiarios. Por ende, lo que intentamos estimar con este indicador es la proporción de la canasta sobre el total del ingreso reportado por el beneficiario. La ecuación es la siguiente: PCAI= Valor de mercado de la CA/Ingreso de los beneficiarios.

Tercero, participación de la Canasta Alimentaria de Banco de Alimentos en el gasto alimentario (PCGA). La encuesta socioeconómica que aplica el banco, también, incluye un reactivo donde se registra el gasto total en alimentación que realiza mensualmente el solicitante del apoyo. Así, con dicha información le añadimos el efecto que tiene la canasta sobre el gasto total en la alimentación del beneficiario. La ecuación es la siguiente: PCGA= Valor de mercado de la CA/ gasto alimentario beneficiarios.

\section{Resultados del análisis nutrimental de la canasta del BAH}

Uno de los objetivos del Banco de Alimentos de Hermosillo (BAH), es el "manejar alimentos a gran escala, obtenidos a través de donativos o compras a bajo costo, para hacerlos llegar a comunidades en pobreza extrema con el fin de mejorar su alimentación”. Lo anterior, con apego a la NOM-169-SSA1-1998, para la asistencia social alimentaria a grupos de riesgo, la cual establece que la ayuda alimentaria deberá aportar como mínimo el 20\% de la ingestión diaria recomendada de energía y proteína para cada grupo beneficiario.

De acuerdo a la NOM-169-SSA1-1998 y con base en los resultados, el aporte nutrimental de la canasta de BAH, cubre el 20\% del aporte de energía, sin embargo, el aporte de proteína queda por debajo de lo establecido, ya que sólo cubre el $17 \%$ de la ingestión diaria recomendada para niños de 3 a 7 años (tabla 1 ). 
Tabla 1

Aporte nutrimental y porcentaje de adecuación (para niños de 3 a 7 años) de la canasta de Banco de Alimentos de Hermosillo.

\begin{tabular}{|l|c|c|c|c|}
\hline Nutrimentos & $\begin{array}{c}\text { Aporte diario por } \\
\text { persona }\end{array}$ & $\begin{array}{c}\text { Recomendación para niños } \\
<7 \text { años }\end{array}$ & $\begin{array}{c}\text { Aporte de la CA del } \\
\text { BAH (\%) }\end{array}$ & $\begin{array}{c}\text { NOM-169- } \\
\text { SSA1-1998 }\end{array}$ \\
\hline Energia (Kcal) & 325 & 1600 & 20 & Bien \\
\hline Proteína (g) & 7 & 40 & 17 & Bajo \\
\hline Fe (mg) & 1.5 & 15 & 10 & No aplica \\
\hline $\begin{array}{l}\text { Vitamina A } \\
\text { (ER) }\end{array}$ & 80.6 & 500 & 16 & No aplica \\
\hline
\end{tabular}

Fuente: elaboración propia con base en información de BAH, (2009).

Además, el aporte de micronutrimientos (hierro y vitamina A) a partir de la canasta del BAH es menor al $20 \%$ de la ingesta diaria recomendada para la población objetivo. La ingesta baja de hierro en la dieta, es un factor de riesgo para el desarrollo de deficiencia nutrimental de este mineral, el cual a su vez se asocia con enfermedades como la anemia. Los efectos de la deficiencia de hierro en edades tempranas persisten por muchos años (Beard et. al., 2001), disminuye la capacidad cognitiva y motora, la atención y memoria .Además, la deficiencia de hierro está asociada con baja productividad aún en tareas que requieren poco esfuerzo disminuyendo la capacidad laboral de las personas (Allen y Guillespie, 2001).

Es necesario tener presente que el aporte nutrimental de la CA, sólo representa una parte de la dieta de las personas beneficiarias. Por lo que se considera importante realizar una evaluación posterior de la ingesta dietaría total, a fin de observar individualmente el aporte nutricional de la CA de $\mathrm{BAH}$ en la dieta de sus beneficiarios.

Sin embargo, empleando el estudio de Ramírez (2002), donde se evaluó la composición nutrimental de la dieta de mujeres migrantes y residentes de la comunidad de Pesquiera, se puede observar que en el consumo diario total de alimentos, el hierro no representa un problema (72 mg). Sin embargo, el consumo diario de vitamina A se encuentra muy por debajo de su ingesta diaria recomendada (87 Equivalentes de Retinol).

Tomando como referencia lo anterior, es posible apreciar la necesidad de incrementar el aporte de vitamina A en la dieta, ya que al existir deficiencia de este micronutrimiento, la integridad de las barreras epiteliales y el sistema inmune, se ven comprometidos antes de que los signos clínicos sean evidentes. Lo que lleva a un aumento en la severidad de las infeccio- 
nes y otros padecimiento asociados al sistema inmune, especialmente en poblaciones vulnerables como los niños (WHO, 1995).

Además de calcular el valor nutrimental de los artículos que componen las canastas, se evaluó el aporte calórico de carbohidratos, lípidos o grasas y proteínas. Así, se encontró que las grasas o lípidos y los carbohidratos representaron la principal fuente nutricional de las canastas con $45 \%$ y $47 \%$ respectivamente, mientras que el contenido proteico solamente se ubicó en 8\% De esta manera, el aporte calórico por lípidos es mucho mayor al recomendado por la WHO/FAO (10-15 \% proteína, 25-30 \% lípidos y 55-60 \% carbohidratos).

\section{Tipificación de la canasta alimentaria}

Para tipificar la canasta alimentaria, se analizaron los menús (alimentos que integran una canasta alimentaria del BAH) de 21 semanas otorgadas a la comunidad de Pesqueira. En lo referente a los productos que integran la canasta, en la Tabla 2 se observa la clasificación de los alimentos que conformaron las CA en básicos, secundarios y periféricos, los cuales se distribuyeron de acuerdo a las frecuencias de consumo.

Tabla 2

Clasificación de los alimentos de la canasta alimentaria

\begin{tabular}{|l|lll|}
\hline Alimentos básicos & Alimentos secundarios & Alimentos periféricos \\
\hline Azúcar & Calabaza & Chayote & Consomé \\
Pasta & Lechuga & Sandía & Café \\
Frijol & Apio & Brócoli & Bebidas Nestlé \\
Arroz & Repollo & Chile Caribe & Latas varias \\
Sardina & Yogurt & Chile poblano & Barra cereal \\
Maicena & Cilantro & Coliflor & Jugos campbell's \\
Tomate & Leche evaporada & Queso Cottage & Bebidas de frutas \\
Cebolla & Nopal & Mango & Nesquick en polvo \\
Chile & Papaya & Toronja & Bebidas nesfrapé \\
Harina/trigo & Ejote & Durazno & Jugos concentrados \\
Pan bimbo & Limón & Melón & Refresco \\
Papa & Zanahoria & Queso & Bebidas saborizadas \\
Harina/maiz & Guayaba & Pepino & Danonino \\
Carne & & & Catsup \\
Leche & & & Salsa picante \\
Tomatillo & & & Gelatina \\
\hline
\end{tabular}

Fuente: elaboración propia con base en información de BAH, (2009). 
A partir de la frecuencia y cantidad de los alimentos, se tipificó la CA del BAH y con base en esto se realizaron recomendaciones para la adecuación nutrimental (tabla 3). La modificación sugiere en función de mejorar el aporte nutricional de la CA en referencia a micronutrimientos. Asimismo, la CA alternativa aporta una menor cantidad de grasa con referencia a la CA promedio que maneja el $\mathrm{BAH}$, por lo que reduce la probabilidad de ser un factor de riesgo en el desarrollo de enfermedades crónicas degenerativas (ECD).

Tabla 3

Canasta recomendada a partir del análisis de los resultados.

\begin{tabular}{|l|l|l|}
\hline Alimentos básicos & Alimentos secundarios & Alimentos periféricos \\
\hline Aceite & Naranja & Avena \\
Azúcar & Calabaza & \\
Pasta & Lechuga* & \\
Frijol & Pepino & \\
Arroz & Papa* & \\
Harina de maíz & Fruta de temporada* & \\
Leche* & & \\
Huevo* & & \\
Cebolla & & \\
Tomate & & \\
Chile* & & \\
\hline
\end{tabular}

*Alimentos no frecuentemente incluidos en la canasta.

Fuente: elaboración propia con base en información de BAH, (2009).

\section{Recomendaciones a la canasta que otorga BAH}

Debido a que el análisis de las CA otorgada por persona demuestra que el aporte de grasas es mayor que lo recomendado, se sugiere que los alimentos con mayor contenido de grasa, se incluyan sólo una vez al mes, limitándose a un producto por CA y no incluir más de dos de estos alimentos en una sola CA. Los alimentos con mayor contenido de grasa fueron: bebidas nestlé, galletas dulces gamesa, nesquick

Procurar la inclusión de alimentos ricos en hierro y vitamina A como son frijol, lenteja, sardina, tomate, zanahoria y frutas y verduras de color rojo, naranja o amarillo, así como lechuga; procurar la inclusión de alimentos con buen aporte de proteína, como son frijol, lenteja, leche, queso, huevo y carne; se recomienda NO INCLUIR en la CA, soda ni bebidas saborizadas, puesto que promueven hábitos alimentarios inadecuados. 


\title{
Análisis económico de los impactos económicos de las canastas alimentarias en los beneficiarios de Pesqueira
}

\author{
Los niveles de Pobreza en Pesqueira
}

Para realizar el análisis de los niveles de pobreza en la localidad, se utilizó la información de las encuestas socio-económicas aplicadas por Banco de Alimentos de Hermosillo y con base en ellas, se clasificó a las familias seleccionadas en los tres niveles de pobreza propuestos por Coneval (2008), como sigue:

Primero, la pobreza alimentaria: Incapacidad para obtener una canasta básica alimentaria, aun si se hiciera uso de todo el ingreso disponible en el hogar para comprar sólo los bienes de dicha canasta (\$548 pesos mensuales por persona para zonas rurales). Segundo, la pobreza de capacidades: Insuficiencia del ingreso disponible para adquirir el valor de la canasta alimentaria y efectuar los gastos necesarios en salud y en educación, aun dedicando el ingreso total de los hogares nada más para estos fines (\$652 pesos mensuales por persona para zonas urbanas y rurales, respectivamente), tercero, la pobreza de patrimonio: Insuficiencia del ingreso disponible para adquirir la canasta alimentaria, así como para realizar los gastos necesarios en salud, vestido, vivienda, transporte y educación, aunque la totalidad del ingreso del hogar sea utilizado exclusivamente para la adquisición de estos bienes y servicios ( $\$ 946,5$ pesos mensuales por persona para zonas urbanas y rurales, respectivamente ${ }^{1}$ ).

De esta manera, el método que se utiliza para analizar los niveles de pobreza es la línea de pobreza per cápita que mide los mínimos de bienestar a los cuales debe acceder una personas para no ser considerado pobre. El método de líneas de pobreza aunque captura varios elementos como alimentación, salud, educación medidas en pesos no es propiamente un análisis multidimensional. Sin embargo, para nuestro cometido, el método de línea de pobreza es adecuado ya que disponemos básicamente de los datos de ingreso y gasto en alimentación.

De las 21 familias que se analizaron sus ingresos y gastos en alimentación, los resultados indican que la pobreza alimentaria afectó alrededor del $90 \%$ de la población, mientras que la pobreza de capacidades al restante $10 \%$; en correspondencia el $100 \%$ del total se situaba en la pobreza de patrimonio. Los datos muestran que la pobreza se encuentra en niveles de los más altos reportados en el país, ya que según datos de CONEVAL (2008), localidades afectadas con alrededor del $70 \%$ del total de la población con pobreza alimentaria significa un grado de marginación alto. Adicionalmente, esto significa que la mayoría de las familias beneficiarias carecen no solamente de los satisfactores más esenciales y básicos como la alimentación, sino que sus oportunidades de educación y salud se acotan de forma alarmante, por ende se corre el riesgo de reproducción del ciclo de la pobreza en la comunidad. 


\section{Cambios en los niveles de pobreza en Pesqueira a partir de la asistencia alimentaria de BAH}

Un supuesto que ha guiado este documento, estriba en la posibilidad de que las Canastas Alimentarias proporcionadas por BAH, contribuyan a reducir los niveles de pobreza de los beneficiarios de Pesqueira. Para poder corroborar dicho supuesto, fue necesario obtener los costos mínimos y máximos que tendrían las canastas si los beneficiarios las adquirirán libremente en los mercados locales. Debido a la heterogeneidad de los productos que compusieron las canastas, se obtuvo que el precio promedio en el mercado sería de \$206, registrando un mínimo de \$152 y un máximo de $\$ 243^{2}$.

Es pertinente aclarar que la variación en cuanto al costo inter-canastas se debió primordialmente a la influencia de los alimentos secundarios y en menor grado de los periféricos, mientras que los básicos mantuvieron una tasa constante del costo de alrededor del 30\% del total. A partir de lo anterior, contrastamos que el ingreso promedio de una familia se incrementaría en $\$ 206$ pesos al mes producto del subsidio obtenido gracias a la canasta, mientras que el porcentaje de sus ingresos que se destinan a alimentación bajaría en 5\%

Aunque a primera vista se nota un incremento en el ingreso de los beneficiarios y una reducción en los gastos destinados a la alimentación respecto al ingreso, aún no estamos en posibilidad de responder si los apoyos del banco efectivamente reducen la intensidad de la pobreza de los beneficiarios.

Para responder la interrogante anterior, elegimos dos vías: en primer lugar utilizamos las medias de gasto en alimentación reportados para cada miembro de las familias apoyadas, la cual arroja un valor de \$278 pesos por persona, cantidad que automáticamente coloca a todos los integrantes en pobreza alimentaria. Sin embargo, puesto que existen beneficiarios individuales o con un número de integrantes menores a la media de 5 personas por familia, es pertinente realizar el análisis beneficiario por beneficiario para obtener mayor congruencia y confiabilidad en los datos.

Al comparar los resultados, se observa una reducción sustantiva en la incidencia de la pobreza alimentaria en $47 \%$ menos al recibir la canasta por parte de BAH. Por su parte, la pobreza de capacidades se incrementa en $4 \%$ y la de patrimonio se reduce en $5 \%$.

La reducción en términos porcentuales de la pobreza alimentaria se debe en gran medida a que la banda que separa a la línea de pobreza de capacidades de la alimentaria es menor (\$104) que el valor del apoyo que otorga BA con las canastas alimentarias (\$293). Es por ello que se reduce la pobreza alimentaria gracias a la transferencia de ingresos por parte del apoyo de BAH, y aumenta la pobreza de capacidades ya que esta refleja el incremento en los ingresos de las familias beneficiarias 
El caso opuesto sucede al comparar la pobreza de capacidades con la de patrimonio, ya que de entrada la banda se amplía a \$294 entre una y otra, factor que incide en el aumento de la primera y la poca variación de la segunda. Es decir, el criterio establecido por Coneval para clasificar a las familias en pobreza de patrimonio en términos económicos supera la transferencia de ingresos que realiza BAH con las canastas.

La variación moderada de la pobreza de patrimonio respecto a la alimentaria radica en la amplitud de la banda de \$398 y sobre todo, en que el promedio del valor de mercado de la canasta de \$293 es significativamente menor a la banda. En términos prácticos los beneficiarios en el límite de pobreza alimentaria con la sola canasta no alcanzan a superar la pobreza de patrimonio al ser la banda mayor que el valor de la canasta que reciben, y por ende, el efecto sólo se vislumbra en mayor grado en la pobreza de capacidades

\section{Participación de las Canastas Alimentarias en los ingresos y gastos de los beneficiarios}

Aunque los resultados presentados en la sección anterior, permiten una visión general del impacto del apoyo de BAH, persiste la interrogante de en ¿Qué grado la canasta de BAH ayuda en términos económicos a los beneficiarios de Pesqueira? En otras palabras, puesto que no todas las familias disponen de la misma cantidad de recursos y tampoco gastan homogéneamente en alimentos, es plausible que los efectos sean diferenciados entre las familias. Para clarificar este punto, se introducen los siguientes dos indicadores: Participación de la Canasta Alimentaria de Banco de Alimentos en el ingreso de los beneficiarios (PCAI) y Participación de la Canasta Alimentaria de Banco de Alimentos en el gasto alimentario (PCGA).

Los valores del primer indicador, apuntan a la categorización de cuatro grupos: el primero comprende a los beneficiarios que en términos de sus ingresos la canasta significó alrededor del 6\% y este agrupa al $28 \%$ del total. El segundo grupo, se encuentra entre 7 y $10 \%$ y representó al 33\%. El tercer y cuarto grupo, comparten valores en términos de porcentajes de beneficiarios aglutinados con 19\% cada uno, mientras que para la PCAI adquieren valores de hasta $16 \%$ y $29 \%$ respectivamente.

Los picos más altos le corresponden al primer y cuarto grupo, lo cual indica que entre los beneficiarios existen diferencias significativas en su nivel de ingreso. A pesar de la disparidad, no se puede corroborar con este solo dato que existan beneficiarios que estuvieran accediendo a las canastas y que sin embargo, su situación no es tan apremiante.

Como se ha expresado a lo largo del documento, las familias son heterogéneas en cuanto al número de integrantes y en los montos destinados a alimentación. Incluso familias que reportan los niveles más altos de ingreso, destinan una parte mucho menor a la media de consumo en alimen- 
tos, por lo que es necesario analizar el segundo indicador para obtener una visión más clara del fenómeno. Los resultados del indicador PCGA se muestran en la tabla 4.

\section{Tabla 4}

\section{Cambios en el gasto alimentario de los beneficiarios al recibir la Canasta}

\begin{tabular}{|c|c|}
\hline $\begin{array}{c}\text { Participación de la CA en el gasto } \\
\text { alimentario (\%) }\end{array}$ & Beneficiarios (\%) \\
\hline 18 & 33 \\
\hline 24 & 33 \\
\hline 29 & 14 \\
\hline 41 & 19 \\
\hline
\end{tabular}

Fuente: elaboración propia con base en información de BAH (2009).

Se observa que los valores de la PCGA son más altos que los de PCAI, ya que los gastos alimentarios en promedio son $50 \%$ menores a los ingresos de las familias, razón que provoca la mayor incidencia de las canastas en su gasto alimentario. Los primeros tres grupos en términos de la PCGA se encuentran separados por un rango no mayor a 6 puntos, y representan casi el $80 \%$ del total. El cuarto grupo es conveniente analizarlo detalladamente debido a que si bien, sólo representa el $20 \%$ del total de la población, su PCGA alcanza un valor muy elevado de $40 \%$. Por lo tanto, es en este grupo donde se expresa el impacto mayor de las canastas y por ende, se configura como la población más vulnerable en términos alimentarios.

Estos resultados, son consistentes aunque en menor escala claro, con los arrojados por otra investigación que analizó los impactos en los niveles de pobreza a nivel agregado de una transferencia adicional de \$170 a los beneficiarios del programa Oportunidades y de $\$ 120$ a las personas no beneficiarias en localidades de menos de 2500 habitantes. Los resultados muestran que dichas transferencias reducirían en 750 mil los pobres generados por las alzas de los alimentos (Cortés, 2008).

En este punto, es preciso abordar con mayor detalle la interrogante que guió esta sección, consistente en analizar el impacto económico de las canastas de BAH en la población beneficiaria de Pesqueira. Con base en los resultados obtenidos se puede afirmar que el impacto es positivo y más allá de las cifras, el ingreso de la población beneficiaria ha aumentado, al disminuir la propensión en gastos de alimentación producto del ingreso que se libera al obtener la canasta. En la misma línea, aunque el impacto de las canastas en los ingresos brutos de las familias es moderado, esto no es así en términos de los gastos alimentarios, ya que las canastas representan 
una parte sustancial del monto destinado a alimentación por parte de la población.

En suma, el impacto es positivo, sobre todo, en los beneficiarios que reportan los montos menores destinados a alimentación. De esta manera, BAH cumple uno de sus objetivos primordiales de ayudar al acceso a los alimentos a poblaciones con extrema pobreza y vulnerabilidad. El siguiente paso, sería corroborar estos resultados a través de encuestas individuales que otorguen un panorama más extenso sobre el destino de los ingresos liberados por las canastas a los beneficiarios.

Una investigación con el enfoque anterior, es sumamente necesaria ya que se infiere que los cambios en los niveles de ingreso y gasto alimentario se configuran de manera automática al integrarse las canastas del banco; pero basta recordar que existen muchos condicionantes como los culturales, ambientales, sociales y políticos que no se han considerado en este análisis y que pueden influir sensiblemente en los resultados arrojados.

\section{Conclusiones}

En las últimas décadas, el concepto de desarrollo local está estrechamente vinculado a la participación de la sociedad civil organizada ya que esta ha comenzado a intervenir activamente en todos los ámbitos que le son posibles, hecho que significa un replanteamiento del papel de todos los actores sociales en una sociedad que evoluciona a pasos agigantados. Durante la primera mitad del siglo pasado el Estado representaba el actor central a partir del cual se generaban las principales iniciativas de crecimiento y desarrollo, sin embargo en las últimas dos décadas transfirió muchas de sus responsabilidades al mercado y a la misma sociedad civil. Además de que esta última ha ido avanzando en la conquista de espacios que estaban bajo el control del gobierno, principalmente en cuestiones de desarrollo.

La emergencia de los actores sociales organizados que ocupan espacios desatendidos por el Estado y el mercado, es un elemento que bien se puede tomar como un indicador del desarrollo de cualquier sociedad. Puesto que pone de manifiesto la capacidad de las personas para hacerle frente a escenarios adversos con una lógica de solidaridad que le corresponde a sociedades modernas y organizadas. Los nuevos espacios públicos ocupados por la sociedad civil, incluye áreas prioritarias como la salud, la alimentación, la pobreza, la seguridad, y el bienestar social en general, y por ello, representan una nueva era de coparticipación social en la resolución de problemas sociales añejos.

Centrando la mirada en nuestro caso de estudio, en particular, se mostró como una organización con sus recursos y limitaciones como BAH intenta combatir el hambre y la desnutrición mediante apoyos alimentarios focalizados a áreas marginadas como Pesqueira. Lo anterior, per se, representa un aspecto notable de la evolución de la sociedad de Hermosillo, y de 
Sonora, sin embargo, la propia orientación del banco hacia la conformación de mejores canastas alimentarias motivó en buena parte este estudio. Así, encontramos que las canastas repartidas por banco de alimentos tienen una capacidad importante aunque insuficiente para copar las necesidades de energía, vitamina A y Hierro por parte de los beneficiarios. En el aspecto económico, las canastas han ayudado a aliviar de manera considerable los gastos en alimentación, y por ello, la pobreza alimentaria de los beneficiarios se ha reducido en cerca del $40 \%$, aunque persiste la pobreza en términos absolutos.

Finalmente, resta decir, que para BAH, este estudio representó una oportunidad para diseñar nuevas estrategias de promoción de donaciones y de adquisición de recursos, bajo la búsqueda de convertirse en una organización que no solamente entrega lo que recibe, sino que es capaz de entregar lo que se requiere. 
Polis, Revista de la Universidad Bolivariana, Volumen 10, $N^{\circ}$ 30, 2011

\section{Notas}

${ }^{1}$ Se utilizó la línea de pobreza de INEGI (2004).

${ }^{2}$ Los precios de los productos se obtuvieron de la página electrónica de Profeco e INEGI, y se tomó el precio mínimo de mercado para evitar sobre estimaciones en el precio de las canastas, ya que parte de los alimentos del banco son donaciones por lo que tomar otro precio, sin duda, repercutiría sustancialmente en la confiabilidad de los cálculos. 


\section{Bibliografía}

Aguilar, L. F. (2008), “Marco para el Análisis de las Políticas Públicas”, Administración \& ciudadanía: revista da Escola Galega de Administración Pública Vol. 3, $N^{\circ}$. 2, EGAP, Santiago de Compostela.

Allen, L., Guillespie, S. (2001), What Works? A review of the efficacy and effectiveness of nutrition interventions. Banco Asiatoco del Desarrollo, ONU, Disponible en: http://www.ifpri.org/sites/default/files/publications/ whatworks.pdf

Ballew, C y Sugerman S. (1996), "Food shopping patterns of low-income Mexican women in Chicago", Ecology of Food and Nutrition $n^{\circ} 35$, Routledge, Londres.

Beard, J. L. (2001), “Iron biology in immune function, muscle metabolism, and neuronal functioning”, The Journal of Nutrition $n^{\circ} 131$, The American Nutrition of Nutritional Sciences, Washington

Boisier, S. (2000), “Desarrollo (local) ¿de qué estamos hablando?”, en Estudios Sociales, Corporación de Promoción Universitaria Chile, Santiago de Chile.

CONEVAL (2008), Informe de evaluación de la política de Desarrollo Social en México, CONVAL, México D.F.

Cortés, F. (2008), Evolución de la pobreza en México y las metas del milenio, (inédito) presentación en Coloquio México y las Metas del Milenio, El Colegio Mexiquense.

Cunill, N. (2006), "Espacios públicos no estatales para mejorar la calidad de las políticas. Una visión sobre América Latina”, en Isunza, E. y Olvera, A. (coords), Democratización, rendición de cuentas y Sociedad Civil: participación ciudadana y control social, CIESAS-Universidad VeracruzanaMiguel Ángel Porrúa, México D.F.

Diamond, J. (2005), Collapse: How societies choose to fail or succeed, Penguin, Londres.

FAO, (2002), Food energy- methods of analysis and conversion factors. FAO, Roma.

Fuentes, C. y Heiss C. (2006), “Sociedad Civil y democracia, ¿Qué podemos aprender de las experiencias de incidencia ciudadana?”, en Varas, A. (ed.), La propuesta ciudadana, Catalonia Editores, Santiago de Chile.

Gore, C. (1984), Regions in Questions Space, Development Theory and Regional Policy, Methen \& Co. Ltd, Londres. 
Holzner, C. (2007), “Voz y voto: participación política y calidad de la democracia en México”, en América Latina Hoy $n^{\circ} 45$, Ediciones Universidad de Salamanca, Salamanca.

Haro Encinas, J. (2008), “Globalización y Salud de los trabajadores. Jornaleros agrícolas y producción de Uva en Pesqueira, Sonora”, en Región y Sociedad, año/vol. XIX, Numero 40, El Colegio de Sonora, Sonora.

Hoyos L. C. (1991), Canasta estatal de consumo de alimentos y aporte de nutrimentos. Tesis de Maestría, Centro de Investigación en Alimentación y Desarrollo A.C., Hermosillo.

López G. (2007), La participación ciudadana en la formulación de planes de desarrollo. Estudio comparado de municipios de Puebla, México y Andalucía, España. Hacia un cambio social en la toma de decisiones, Factoría de Ideas, Centro de Estudios Andaluces, Sevilla.

Mouzelis, N. (1991), Organización y Burocracia, Ediciones 62, Madrid.

Olson J. A. (1987), "Recommended dietary intakes (RDI) of vitamin A in humans”, en The American Journal of Clinical Nutrition Vol 45, Washington.

OMS (1995), Global Prevalence of Vitamin A Deficiency. Working Paper $\mathrm{n}^{\circ}$ 2, OMS. Disponible en: http://whqlibdoc.who.int/publications/2009/ 9789241598019_eng.pdf

Ramírez, G. (2002), El efecto del proceso de migración en el estado nutricio de familias jornaleras migrantes en el noroeste de México. Tesis del Centro de Investigación en Alimentación y Desarrollo, A.C., Hermosillo.

Vazquez Barquero, A. (2005), Las Nuevas Fuerzas del Desarrollo, Antonio Bosh Editor, Barcelona.

Recibido: 16.08.2011

Aceptado: 23.11.2011 